Check for updates

Cite this: RSC Adv., 2018, 8, 36769

\title{
Basic ionic liquid as catalyst and surfactant: green synthesis of quinazolinone in aqueous media
}

\begin{abstract}
Yaping Zhang, (D) *acd Bin Zhen, ${ }^{\text {bc }}$ Hansheng Li (iD ${ }^{\mathrm{c}}$ and Yaqing Feng ${ }^{\text {ad }}$
Basic imidazolium-based ionic liquids not only possess the extraordinary physicochemical properties of ionic liquids, but also have excellent basicity and surfactivity. 1-Propyl-3-alkylimidazole hydroxide ionic liquids ([PRIm] $[\mathrm{OH}])$ were synthesized and their catalytic and surfactant behavior were studied in this work. [PRIm] $[\mathrm{OH}]$ owned excellent surfactivity, and their alkyl chains and ion pairs benefit hydrophobicity and hydrophilicity respectively. The surfactivity of $[\mathrm{PR} / \mathrm{m}][\mathrm{OH}]$ increased with increasing alkyl chain length. [PRIm] $[\mathrm{OH}]$ showed better catalytic performance than $\mathrm{NaOH}$ in the condensation of 2aminobenzonitrile with cyclohexanone in aqueous medium, and the catalytic performance was well coincident with their surfactant behavior. [PRIm] [OH] could decrease the interfacial tension of solvent effectively and form micelles in water. The formed micelles could solubilise more reactants into water and effectively increase the chance of contact between reactants and catalytic active sites. The catalyst dosage obviously affected catalytic performance. The catalytic system is a promising recyclable system.
\end{abstract}

Received 29th July 2018

Accepted 25th October 2018

DOI: $10.1039 / \mathrm{c} 8 \mathrm{ra06378h}$

rsc.li/rsc-advances

Imidazolium-based ionic liquids are an important kind of

\section{Introduction}

Ionic liquids have attracted extensive attention in a variety of fields owing to their extraordinary physicochemical properties, such as wide liquid range, high thermal stability, powerful solvent capacity, nonflammability, nonvolatility, and good reusability. They can work as catalysts, environmentally-benign solvents, green reaction media, electrolytes and surfactants in various fields like organic synthesis, ${ }^{1}$ catalytic chemistry, ${ }^{2,3}$ electrochemistry, ${ }^{4}$ separation, ${ }^{5}$ material preparation ${ }^{6}$ and electrophoretic displays. ${ }^{7,8}$ Moreover, their properties can be varied to some extent for specific applications by changing their structure. ${ }^{9}$

Although significant progress has been achieved in the study of acidic and neutral ionic liquids, the reports on basic ionic liquids are relatively rare. Basic ionic liquids have been reported to promote reactions, such as Michael addition, ${ }^{1}$ Markovnikov addition ${ }^{10}$ and Knoevenagel condensation, ${ }^{11}$ and Henry reaction, ${ }^{12}$ and they have shown good catalytic efficiency and recycling property. However, much attention has been focused on their significant roles in controlling various organic reactions as solvents and catalysts. Our understanding of how basic ionic liquids control the desired reactions is very limited.

${ }^{a}$ School of Chemical Engineering and Technology, Tianjin University, Tianjin 300072, China. E-mail: zhypin126@126.com

${ }^{b}$ College of Chemistry and Chemical Engineering, Tianjin University of Technology, Tianjin, 300384, China. E-mail: zhenbin00000@163.com

${ }^{c}$ School of Chemistry and Chemical Engineering, Beijing Institute of Technology, Beijing, 102488, China

${ }^{d}$ Collaborative Innovation Center of Chemical Science and Engineering (Tianjin), Tianjin 300072, China ionic liquid, and they usually consist of ion pairs and long alkyl chains. ${ }^{713}$ These ionic liquids with long alkyl chains could aggregate together and form micelles in aqueous solution, which favors the miscibility of immiscible substances due to the solubilization effect of the micelles. ${ }^{14-18}$ For basic imidazoliumbased ionic liquids, they could own both basicity and surfactivity.

Quinazolinones are important and useful nitrogencontaining heterocyclic compounds and show diverse bioactivities, including anti-bacterial, anti-inflammatory, antitumor and anti-hypertensive. ${ }^{19-22}$ The traditional methods to synthesize quinazolinones have many drawbacks like multistep procedures, high cost, toxic reagents, harsh reaction conditions and poor yields. ${ }^{23-28} \mathrm{~A}$ condensation between 2aminobenzonitrile and carbonyl compounds was reported to synthesize quinazolinones, ${ }^{29}$ and alkaline catalysts, typically $\mathrm{NaOH}$ or 1,8-diazabicyclo [5.4.0] undec-7-ene(DBU), are used to promote this reaction. However, high corrosivity and difficult recovery hinder the wide application. $\mathrm{N}$-heterocyclic carbine was used to catalyze in this reaction by our group, and the yield of quinazolinone arrived $97 \% .{ }^{16}$ Unfortunately, N-heterocyclic carbene catalyst was easy to decompose and difficult to be recycled. The aqueous solution of ionic liquid with both basicity and amphiphilicity is potential efficient, green and recoverable catalytic system for the synthesis of quinazolinones.

In this work, basic 1-propyl-3-alkylimidazolium hydroxide ionic liquids ([PRIm][OH]) were synthesized and used to promote the synthesis of quinazolinone in aqueous medium. The influences of surfactant behavior and catalytic 
performance of the synthesized ionic liquids on this reaction were investigated.

\section{Experimental}

\subsection{Materials and reagents}

1-Propylimidazole (98\%) was purchased from Energy Chemical. 1-Bromopropane (99\%), 1-bromohexane ( $\geq 98 \%)$, 1-bromododecane (99\%) and 1-bromohexadecane (99\%) were purchased from Sinopharm Chemical Reagent Beijing Co. Ltd. Absolute ether, hydrochloric acid, hydrogen nitrate, $\mathrm{Na}_{2} \mathrm{CO}_{3} \mathrm{NaOH}$, and $\mathrm{AgNO}_{3}$ were analytically pure and purchased from Beijing Chemical Works. Anion exchange resin, 2-aminobenzonitrile and cyclohexanone were purchased from J\&K Scientific Ltd. Water used was distilled water.

\subsection{Synthesis of basic ionic liquids}

[PRIm][Br] (R: propyl, hexyl, dodecyl or hexadecyl) ionic liquids were prepared through quaterisation. Equimolecular 1-propylimidazole and 1-bromoalkane (1-bromopropane, 1-bromohexane, 1-bromododecane or 1-bromohexadecane) were mixed in a three-necked flask and stirred at $60{ }^{\circ} \mathrm{C}$ for $24 \mathrm{~h}$. After reaction, the mixture was washed with absolute ether for five times for the purification of [PRIm] [Br] (R: propyl, hexyl, dodecyl). For $[\mathrm{PHeIm}][\mathrm{Br}]$, the washing process was carried out under $-4{ }^{\circ} \mathrm{C}$, at which $[\mathrm{PHeIm}][\mathrm{Br}]$ would change into solid phase to facilitate the separation of ionic liquid and solvent. Then [PRIm] $\mathrm{Br}]$ was dried at $80^{\circ} \mathrm{C}$ for $24 \mathrm{~h}$ to achieve the final ionic liquids. Finally, an aqueous solution of [PRIm] $\mathrm{Br}]\left(0.1 \mathrm{~g} \mathrm{~mL}^{-1}\right)$ was then passed through a column filled with strong basic anion exchange resin to obtain an aqueous solution of $[\mathrm{PRIm}][\mathrm{OH}] . \mathrm{HNO}_{3} / \mathrm{AgNO}_{3}$ aqueous solution was used for judging of final completion of ion exchange.

The $[\mathrm{PRIm}][\mathrm{OH}]$ concentration of the obtained aqueous solution were tested by neutralization titration using hydrochloric acid solution. Then a series of aqueous solution of $[\mathrm{PRIm}][\mathrm{OH}]$ with various concentration were prepared and used in the following experiments and tests.

\subsection{The condensation of 2 -aminobenzonitrile with cyclohexanone}

The condensation reaction was performed at $60{ }^{\circ} \mathrm{C}$ in an aqueous solution of [PRIm] $[\mathrm{OH}]$ (or $\mathrm{NaOH})$, which contained $0.4 \mathrm{mmol}$ [PRIm][OH] (or $\mathrm{NaOH}$ ), $1.0 \mathrm{mmol}$ 2-aminobenzonitrile and $1.5 \mathrm{mmol}$ cyclohexanone (Scheme 1). The yield of quinazolinone was determined by a Techcomp LC2000 HPLC using an ultraviolet detector. A Kromasil 100-5 C18 column $(4.6 \mathrm{~mm} \times 5 \mathrm{~m} \times 250 \mathrm{~mm})$ was used and the mobile phase was

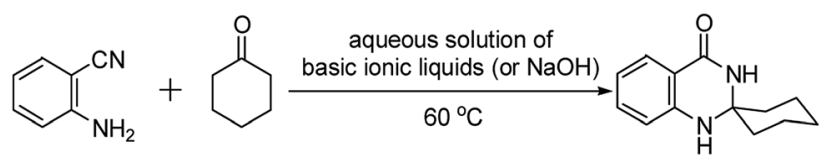

Scheme 1 Condensation of cyclohexanone with 2aminobenzonitrile. a weakly basic water/methanol mixture $(50: 50 \mathrm{v} / \mathrm{v})$ at a flow rate

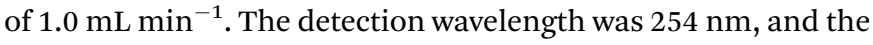
column was held at room temperature.

\subsection{Characterization}

Fourier transform infrared (FTIR) spectrometry analysis was carried out on a NICOLET iS10 Fourier transform infrared spectrometer (Thermo, America) in a frequency range of 400$4000 \mathrm{~cm}^{-1}$ with a resolution of $4 \mathrm{~cm}^{-1}$, a scanning number of 16 and $\mathrm{KBr}$ as a reference. ${ }^{1} \mathrm{H}$ NMR spectra of [PRIm] $[\mathrm{Br}]$ were tested on a varian mercury-plus $400 \mathrm{MHz}$ nuclear magnetic resonance spectrometer (Varian, America) using $\mathrm{CDCl}_{3}$ as solvent, and ${ }^{1} \mathrm{H}$ NMR spectra of [PRIm] [OH] were performed on a Ascend $400 \mathrm{MHz}$ nuclear magnetic resonance spectrometer using $\mathrm{D}_{2} \mathrm{O}$ as solvent. Surface tension values of aqueous [PRIm] $[\mathrm{OH}]$ solution were tested on an QBZY automatic surface tension instrument (Shanghai FangRui Instrument CO, LTD, China) by Wilhelmy plate method. The instrument was adjusted by standard weight before test, and distilled water was used as test calibrant. Each solution was stirred to ensure the homogeneous mixing, and then was injected to a beaker with doublewall casing. Water from a water bath was pumped round the double walled casing with the aid of a SYC-1015D thermostat (Gongyi City Yuhua Instrument CO, LTR, China), the temperature of which was kept constant within $\pm 0.1{ }^{\circ} \mathrm{C}$ of a desired temperature. The surface tension measurements were carried out at $60^{\circ} \mathrm{C}$ and the measurements were repeated three times at each step. A FE30 conductivity meter (Mettler Toledo, Switzerland) was used to test conductivity values of aqueous [PRIm] $[\mathrm{OH}]$ solution. The current source was DC9v and the accuracy level was 0.5. A series of aqueous [PRIm] $[\mathrm{OH}]$ solution with various concentration were prepared in advance and tested at $15{ }^{\circ} \mathrm{C}$. The measurements were repeated three times. The size analyses of ionic liquid micelles were carried out using a Nano ZS particle size \& zeta potential analyzer (Malvern, England) at $25{ }^{\circ} \mathrm{C}$. A series of aqueous solution of $[\mathrm{PRIm}][\mathrm{OH}]$ were prepared in advance and tested at least three times for each sample.

\section{Results and discussion}

Chemical composition of the synthesized [PRIm] [Br] ionic liquids were analyzed by FTIR and ${ }^{1} \mathrm{H}$ NMR spectra, and shown in Fig. 1. As shown in Fig. 1(a), $2960 \mathrm{~cm}^{-1}, 2925 \mathrm{~cm}^{-1}$ and $2854 \mathrm{~cm}^{-1}$ were assigned to stretching vibration of saturated $\mathrm{C}-\mathrm{H}$ bond; $1464 \mathrm{~cm}^{-1}$ was assigned to bending vibration of saturated $\mathrm{C}-\mathrm{H}$ bond; $1378 \mathrm{~cm}^{-1}$ was assigned to the symmetrical bending vibration of $\mathrm{CH}_{3} ; 721 \mathrm{~cm}^{-1}$ was assigned to swing vibration of alkyl chain of $\left(\mathrm{CH}_{2}\right)_{n}(n \geq 4)$; at the same time, $3062 \mathrm{~cm}^{-1}$ and $3130 \mathrm{~cm}^{-1}$ were assigned to vibration of unsaturated $\mathrm{C}-\mathrm{H}$ bond; $1563 \mathrm{~cm}^{-1}$ was assigned to stretching vibration of imidazole ring, $750 \mathrm{~cm}^{-1}$ was assigned to the bending vibration of imidazole ring. Besides, all of the hydrogen atoms appeared in their ${ }^{1} \mathrm{H}$ NMR spectra (Fig. 1(b)). The analyses above prove the formation of $[\mathrm{PRIm}][\mathrm{Br}]$ ionic liquid. 

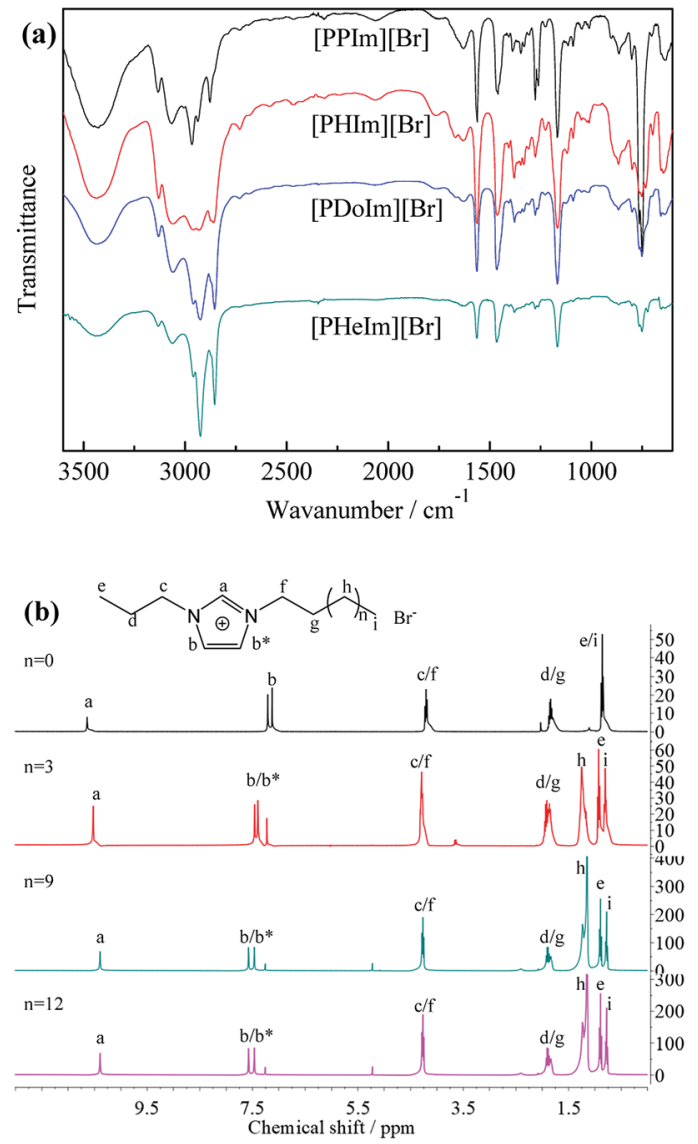

Fig. 1 FTIR (a) and ${ }^{1} \mathrm{H}$ NMR (b) spectra of [PRIm][Br].

After the ion-exchange the $[\mathrm{PRIm}][\mathrm{OH}]$ aqueous solutions were obtained and their ${ }^{1} \mathrm{H}$ NMR spectra were shown in Fig. 2. Compared with the ${ }^{1} \mathrm{H}$ NMR spectra of [PRIm] [Br], all hydrogens in cations showed up. However, the hydrogen assigned to $\mathrm{N}-$ $\mathrm{CH}-\mathrm{N}$ of imidazole ring was not so obvious and the peak assigned to $\mathrm{OH}^{-}$did not appear, which were due to the rapid exchange of that $\mathrm{H}$ atom with $\mathrm{D}$ in $\mathrm{D}_{2} \mathrm{O}$ solvent on the NMR time scale. ${ }^{14}$ It could be seen that the cations in [PRIm $][\mathrm{OH}]$ preserve their structure after the ion exchange process. Combined with the disappearance of $\mathrm{Br}^{-}$judged by $\mathrm{HNO}_{3} / \mathrm{AgNO}_{3}$ aqueous

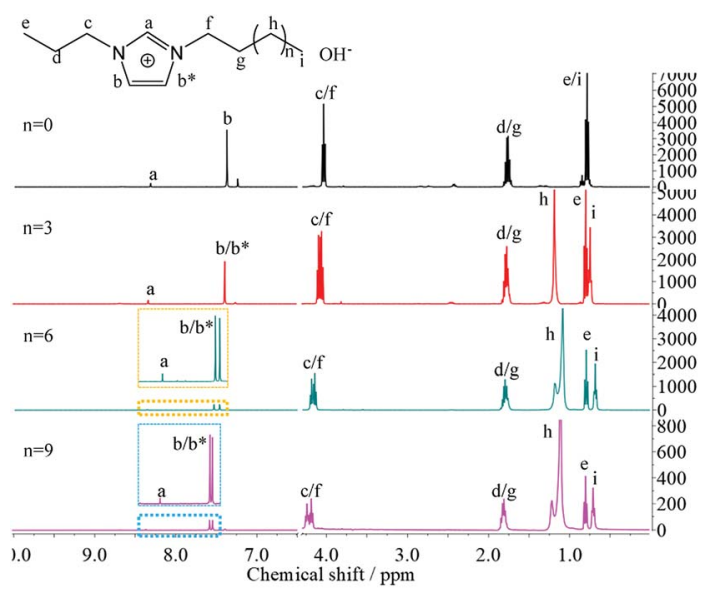

Fig. $2{ }^{1} \mathrm{H}$ NMR spectra of [PRIm] $[\mathrm{OH}]$. solution, it could be concluded that the formation of [PRIm] $[\mathrm{OH}]$.

The synthesized ionic liquids owned hydrophilic ion pairs and hydrophobic alkyl groups, endowing them with amphipathy. The obtained [PRIm] [Br] and [PRIm] [OH] could be dissolved in water easily, indicating their excellent hydrophilicity. Besides, in the FTIR spectra of $[\mathrm{PRIm}][\mathrm{Br}]$, the peaks of $3435 \mathrm{~cm}^{-1}$ and $1630 \mathrm{~cm}^{-1}$ assigned to the stretching vibration and bending vibration of $\mathrm{O}-\mathrm{H}$ bond of absorbed water also showed up, indicating the existence of absorbed water. It was due to that [PRIm] [Br] were apt to absorb water very easily. Nevertheless, with the alkyl chain length increasing, the intensities of these peaks decreased, meaning that the tendency of absorbing water decreased. It proved that the alkyl group favors the hydrophobicity of ionic liquids, and with the alkyl chain length increasing, the hydrophobicity of ionic liquids increases.

The amphipathic structure of $[\mathrm{PRIm}][\mathrm{OH}]$ provides the possibility of their surfactivity. Micellization is an important characterization of the surfactants, and critical micelle concentration $(\mathrm{CMC})$ of $[\mathrm{PHIm}][\mathrm{Br}]$ and [PDoIm $][\mathrm{Br}]$ were tested by conductance method and compared with the reported values. As shown in Fig. 3 and Table 1, the CMC values of the ionic liquids at $15{ }^{\circ} \mathrm{C}$ were close to the reported ionic liquids. The CMC value of [PDoIm $][\mathrm{Br}]$ was derived by the equation descripted as $\log (\mathrm{CMC})=A-B N,{ }^{30}$ where $N$ is number of carbon atoms in the chain, $A$ and $B$ are constant determined from known values of CMC for one member of the series. The calculated CMC value of [PDoIm] $[\mathrm{Br}]$ was between the CMC values of 1-methyl-3-dodecylimidazolium bromide ([MDoIm] [Br]) and 1-methyl-3-tertadecylimidazolium bromide ([MTeIm] [Br]) at $15{ }^{\circ} \mathrm{C}$, which were reported to be $10.1 \mathrm{mmol} \mathrm{L}^{-1}$ and $2.7 \mathrm{mmol} \mathrm{L}^{-1}$ respectively. ${ }^{31}$ This proved the accuracy of the
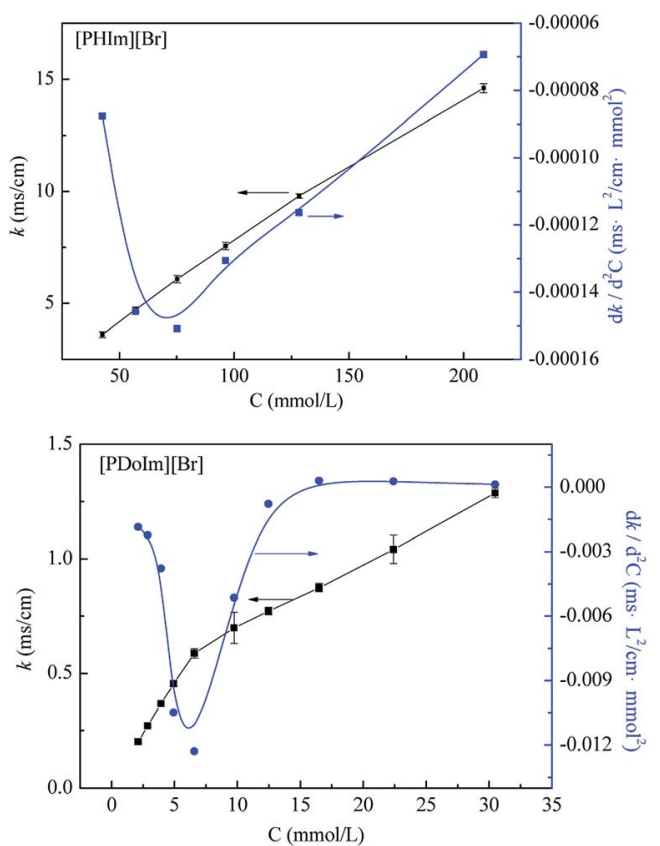

Fig. 3 Plots of conductance against the concentration of ionic liquid aqueous solution. 
Table $1 \mathrm{CMC}$ values of $[\mathrm{PRIm}][\mathrm{Br}]$. The test temperature: $15^{\circ} \mathrm{C}$

\begin{tabular}{lc}
\hline Ionic liquids & CMC $\left(\mathrm{mmol} \mathrm{L}^{-1}\right)$ \\
\hline$[\mathrm{PPIm}][\mathrm{Br}]^{a}$ & 239.2 \\
{$[\mathrm{PHIm}][\mathrm{Br}]^{b}$} & 70.4 \\
{$[\mathrm{PDoIm}][\mathrm{Br}]^{b}$} & 6.1 \\
{$[\mathrm{PHeIm}][\mathrm{Br}]^{a}$} & 1.2 \\
{$[\mathrm{MDoIm}][\mathrm{Br}]^{c}$} & 10.1 \\
{$[\mathrm{MTeIm}][\mathrm{Br}]^{c}$} & 2.7 \\
${ }^{a}$ Calculated values. ${ }^{b}$ Tested values. $^{c}$ Reported values from ref. 31.
\end{tabular}

derivation. The [PRIm] $[\mathrm{Br}]$ gave $\mathrm{CMC}$ values, and with carbon numbers of the alkyl groups increasing, the CMC values of [PRIm] $[\mathrm{Br}]$ decreased greatly. It could be concluded that the synthesized [PRIm] [Br] own surfactivities, and their surfactivities increased with the alkyl chain length increasing.

Surface tension is another powerful tool to gain insight into the surface activity of surfactants at various surfaces and interfaces. The surface tension values of the aqueous solution of $[\mathrm{PRIm}][\mathrm{OH}]$ as a function of their concentration at $60{ }^{\circ} \mathrm{C}$ were tested by Wilhelmy plate method and shown in Fig. 4(a). The surface tension value of water tested at $60{ }^{\circ} \mathrm{C}$ is $66.28 \mathrm{mN} \mathrm{m}^{-1}$, which is in good agreement with the reported value of water by Vargaftik et al. $^{32}$ The surface tension value of aqueous solution of $\mathrm{NaOH}$ is $48.10 \mathrm{mN} \mathrm{m}^{-1}$. Compared with aqueous solution of $\mathrm{NaOH}$, the surface tension value of aqueous solution of [PRIm] $[\mathrm{OH}]$ decreased rapidly, and the surface tension deviation increased obviously with alkyl chain length increasing. It indicates that $[\mathrm{PRIm}][\mathrm{OH}]$ own excellent surfactivity, and the surfactivity increased obviously with alkyl chain length increasing. Dynamic light scattering has been employed to gain insight into the size of the formed micelles. Fig. 4(b) shows the light scattering profiles for $[\mathrm{PRIm}][\mathrm{OH}]$ at a concentration three times that of their respective cmc values. It can be seen in Fig. 4(b) that the formed micelles exhibit hydrodynamic diameters, proving the existence of micelles.

The surfactant behavior and catalytic performance of basic ionic liquid were characterized by the model condensation of 2aminobenzonitrile with cyclohexanone and displayed in Fig. 5. It could be observed that $\mathrm{NaOH}$ showed the lowest catalytic performance with the product yield of $5 \%$. Compared with $\mathrm{NaOH},[\mathrm{PPIm}][\mathrm{OH}]$ showed a slightly better catalytic property. For basic ionic liquids with carbon numbers of the alkyl groups no more than 12 , the catalytic performance increased obviously with alkyl chain length increasing, while for $[\mathrm{PHeIm}][\mathrm{OH}]$ whose carbon number of alkyl group exceed 12 , its catalytic performance decreased slightly. [PDoIm] [OH] achieved the highest product yield. The analyses above indicated the significant role of $[\mathrm{PRIm}] \mathrm{OH}$ in the condensation of 2-aminobenzonitrile with cyclohexanone. The reaction process was observed and displayed in Fig. 6. Basic [PDoIm] $[\mathrm{OH}]$ aqueous solution was completely colorless transparent. When the hydrophobic reactants were added into the solution, a yellow and transparent solution formed. With the reaction going on, white solid product formed and precipitated at the bottom till the reaction ended.
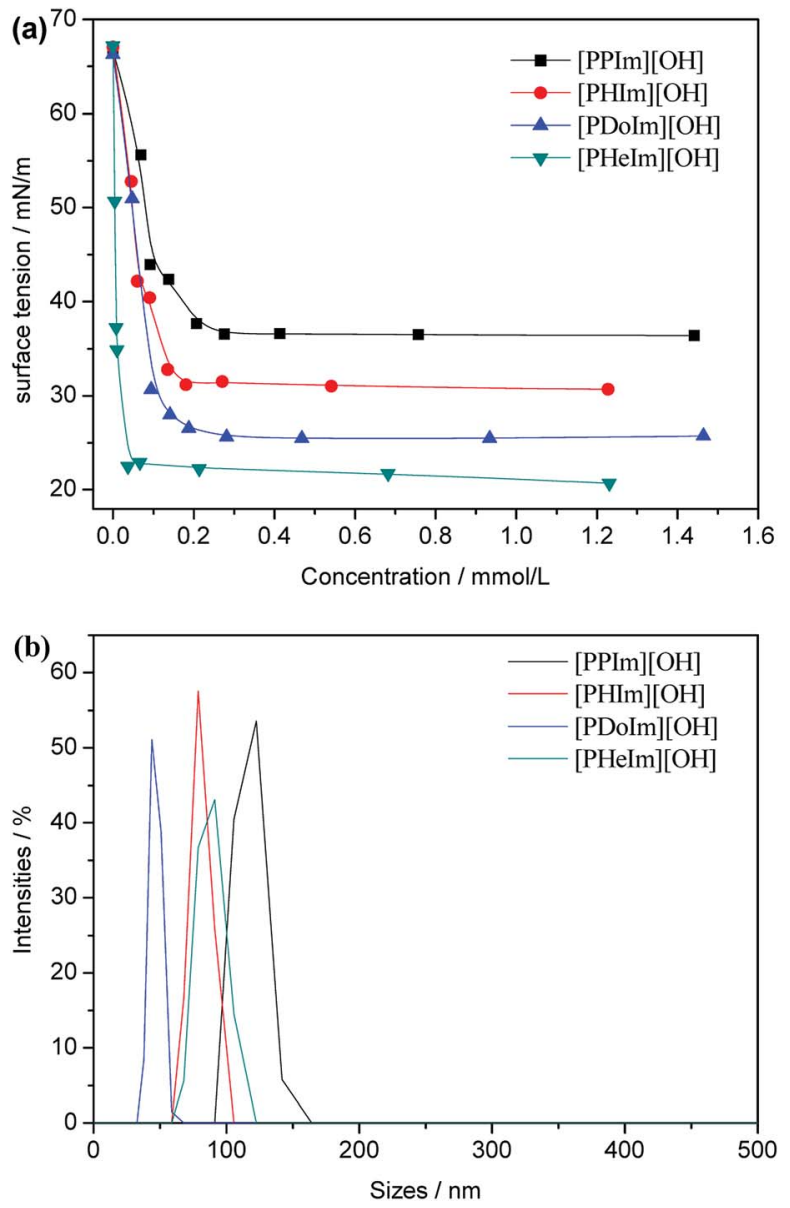

Fig. 4 (a) Variation of surface tension in aqueous solutions of [PRIm] $[\mathrm{OH}]$ as a function of their concentration at $60{ }^{\circ} \mathrm{C}$; (b) dynamic light scattering profiles represented as intensity $\%$ for micellar solutions of $[P R I m][O H]$ showing different hydrodynamic diameters at $25^{\circ} \mathrm{C}$.

To reveal the disperse state of each component and analyze synthesis mechanism, the aggregation states of [PRIm] $[\mathrm{OH}]$ and reactants in water were simulated by dissipative particle dynamics method. As shown in Fig. 7(a), [PDoIm] [OH] with

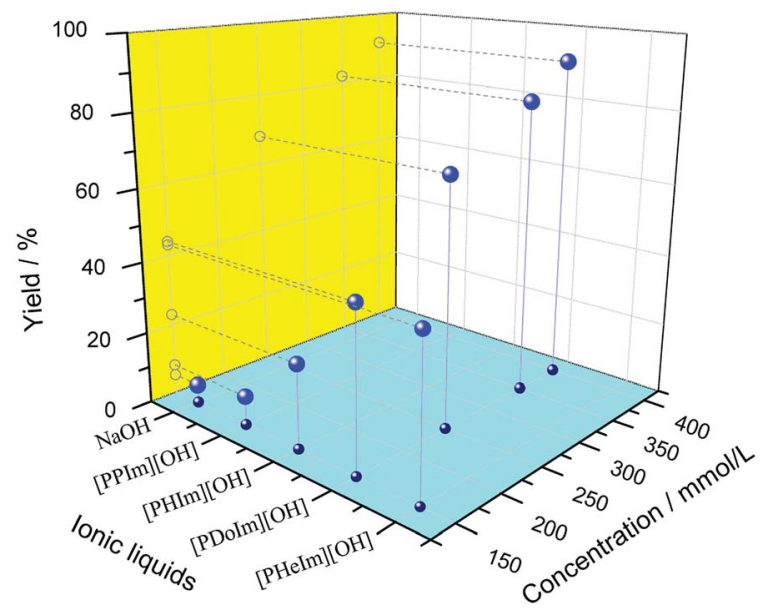

Fig. 5 Catalytic performance of $[\mathrm{PRIm}][\mathrm{OH}]$. The reaction time: $120 \mathrm{~min}$. 


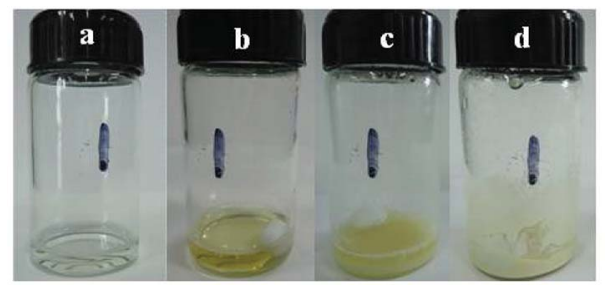

Fig. 6 Photos of the condensation process, (a) aqueous solution of [PDolm] $[\mathrm{OH}]$; (b) aqueous solution of [PDolm] $[\mathrm{OH}]$ with reactants; (c) reaction system after $30 \mathrm{~min}$; (d) reaction system at the end.

concentration of $150 \mathrm{mmol} \mathrm{L}^{-1}$ could be dissolved in water and formed a transparent solution at $60^{\circ} \mathrm{C}$. Cyclohexanone could be dissolved in water at $60{ }^{\circ} \mathrm{C}$, while 2-aminobenzonitrile showed poor miscibility with water (Fig. 7(b)). When [PDoIm][OH] and reactants were added into water simultaneously, an uniform transparent liquid formed (Fig. 7(c) and 6(b)). The snapshot of the simulated system showed that $[\mathrm{PDoIm}][\mathrm{OH}]$ mainly aggregated on the interface between water and hydrophobic reactant and the micelles formed. At one hand, the formed micelles brought increased contact interface area and contact odds; at the other hand, the basic catalysts at the interface facilitated the contact of reactants with catalytic activity sites and accelerated reaction process. Furthermore, the formed solid product would precipitate from the reaction system (Fig. 6(c) and (d)), which could also favor reaction conversion.

The effect of catalyst dosage on the condensation was investigated and illustrated in Fig. 5. It was obvious that the reaction rate increased sharply with the $[\mathrm{PDoIm}][\mathrm{OH}]$ concentration increasing. The states of the components in the reaction systems were simulated and shown in Fig. 7(c-f). As the [PDoIm] $[\mathrm{OH}]$ concentration increased from $150 \mathrm{mmol} \mathrm{L}^{-1}$ to $400 \mathrm{mmol}$ $\mathrm{L}^{-1}$, more $[\mathrm{PDoIm}][\mathrm{OH}]$ assembled together, and the size of the formed micelles increased, resulting in more solubilization of hydrophobic reactants in the "micro micelle reactor" and more catalytic sites at the interface of the micelles.

Based on the analyses above, basic ionic liquids play a key role in the synthesis of quinazolinone in aqueous media, and

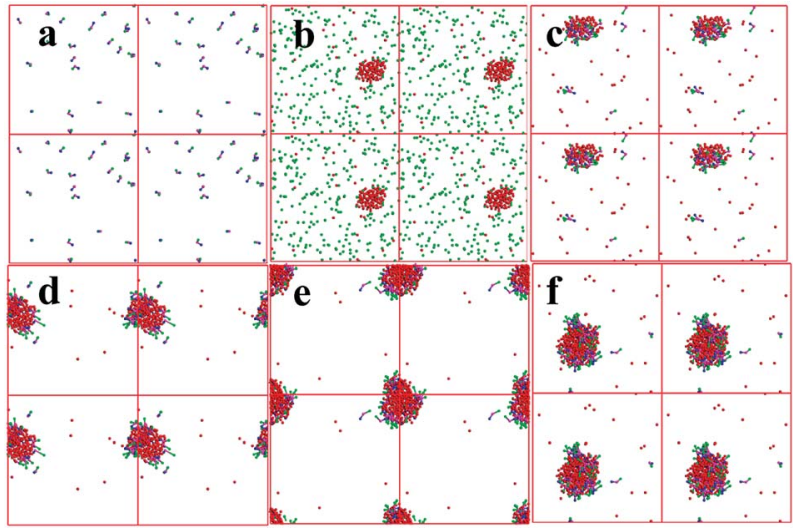

Fig. 7 Snapshots of simulated systems of [PDolm] [OH] in water (a), reactants in water $(b)$, reaction systems with $[P D o l m][O H]$ dosages of $150 \mathrm{mmol} \mathrm{L}^{-1}$ (c), $250 \mathrm{mmol} \mathrm{L}^{-1}$ (d), $350 \mathrm{mmol} \mathrm{L}^{-1}$ (e) and $400 \mathrm{mmol}$ $\mathrm{L}^{-1}(\mathrm{f})$.

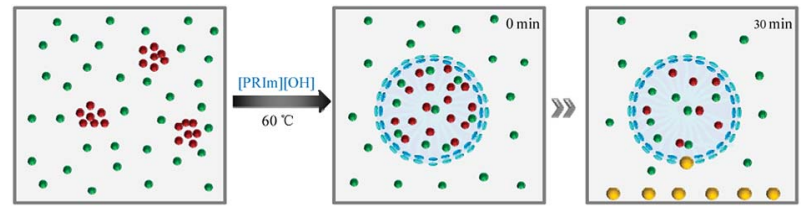

Scheme 2 Synthesis scheme of the condensation. Red, green and yellow spheres represented respectively 2 -aminobenzonitrile, cyclohexanone and product, and ellipsoid pairs with long tails are basic ionic liquid.

Table 2 Recyclable property of [PDolm] $[\mathrm{OH}]^{a}$

\begin{tabular}{ll}
\hline Catalysts & $Y(\%)$ \\
\hline$[\mathrm{PDoIm}][\mathrm{OH}] 1^{\text {st }}$ & 68 \\
{$[\mathrm{PDoIm}][\mathrm{OH}] 2^{\text {nd }}$} & 66 \\
{$[\mathrm{PDoIm}][\mathrm{OH}] 3^{\text {rd }}$} & 66 \\
${ }^{a}$ Reaction time: $240 \mathrm{~min}$, catalyst: $150 \mathrm{mmol} \mathrm{L}^{-1}$. &
\end{tabular}

their basicity and surfactivity favor the condensation profoundly (Scheme 2). When $\mathrm{NaOH}$ was used as catalyst, hydrophobic reactants could not be dissolved in water, and the reaction could only happen at the limited interface of water and reactants. The limited interface supplied insufficient contact of reactants with catalytic active sites, resulting in a poor product yield. When [PPIm] $[\mathrm{OH}]$ was used as catalyst, its concentration in the reaction system was $150 \mathrm{mmol} \mathrm{L}^{-1}$, much lower than its CMC value of $546 \mathrm{mmol} \mathrm{L}^{-1}$, which affected the miscibility of the reactants in water badly and resulted in poor product yield. For [PRIm $][\mathrm{OH}](\mathrm{R}=\mathrm{H}$, Do and $\mathrm{He})$ ionic liquids, which showed excellent surfactant, their dosages in the reaction system were higher than their CMC values. When they were added into the reaction system, they could shrink the interfacial tension greatly and form micelles. The formed micelles could solubilise more reactants into water and enlarge the contact interface and chance of reactants with catalytic active sites effectively, leading to increased product yield. As increasing of their dosages, the solubilization was definitely improved, and the product yield increased remarkably. The catalytic performances of basic ionic liquids are well coincidence with their surfactant behavior.

Keeping as close as possible to the general experimental conditions stated above, the recyclable property of [PDoIm $][\mathrm{OH}]$ was studied and illustrated in Table 2. The aqueous solution of [PDoIm $][\mathrm{OH}]$ was collected by simple filtration and reused in the reaction. It could be seen that the product yield decreased in the second run, which was probably owing to the wastage of [PDoIm $][\mathrm{OH}]$ caused by the adsorption onto the solid product of quinazolinone at the first run. In the third run, the yield of quinazolinone did not change obviously. This result indicated that the catalytic system is a promising recyclable system.

\section{Conclusions}

The basic [PRIm] [OH] ionic liquids were synthesized via quaterisation and ion exchange process. [PRIm] $[\mathrm{OH}]$ owned 
hydrophilic ion pairs and hydrophobic alkyl groups, endowing them with excellent surfactivity. The surfactivity of [PRIm][OH] increased with alkyl chain length increasing. The synthesized [PRIm] [OH] were used to promote the condensation of 2-aminobenzonitrile with cyclohexanone in aqueous medium. Compared with $\mathrm{NaOH},[\mathrm{PRIm}][\mathrm{OH}]$ showed better catalytic property, and the tendency was very coincidence with the surfactant behaviour. The surfactivity of basic ionic liquid could decrease the interfacial tension greatly and help to form micelles. The formed micelles could solubilise more reactants into water, and enlarge the contact interface and the contact chance of reactants with catalytic active sites effectively. Higher catalyst dosage favored the catalytic performance, and the best catalytic performance was achieved at $400 \mathrm{mmol} \mathrm{L}^{-1}$. The catalytic system is a promising recyclable system.

\section{Conflicts of interest}

There are no conflicts to declare.

\section{Acknowledgements}

This work was supported by the National Natural Science Foundation of China (Grant No. 20976013 and 21706185).

\section{References}

1 B. C. Ranu and S. Banerjee, Org. Lett., 2005, 7, 3049.

2 Q. Zhang, S. Zhang and Y. Deng, Green Chem., 2011, 13, 2619.

3 Y. Zhang, Q. Jiao, B. Zhen, Q. Wu and H. Li, Appl. Catal., A, 2013, 453, 327.

4 P. K. Chhotaray, S. Jella and R. L. Gardas, J. Chem. Thermodyn., 2014, 74, 255.

5 A. Pinkert, K. N. Marsh, S. Pang and M. Staiger, Chem. Rev., 2009, 109, 6712.

6 G. Singh, M. Kaur, M. Drechsler and T. S. Kang, Chem. Commun., 2018, 54, 2432-2435.

7 Y. Zhang, B. Zhen, S. A. S. Al-Shujaa, G. Zhou, X. Li and Y. Feng, Dyes Pigm., 2018, 148, 270.

8 Y. Zhang, B. Zhen, R. Li, S. Meng, X. Li and Y. Feng, Mater. Lett., 2018, 211, 17.

9 Z. Li, H. Wang, M. Chu, P. Guan, Y. Zhao, Y. Zhao and J. Wang, RSC Adv., 2017, 7(71), 44688-44695.
10 J. Xu, B. Liu, W. Wu, C. Qian, Q. Wu and X. Lin, J. Org. Chem., 2006, 71, 3991.

11 X. Chen, X. Li, H. Song, Y. Lu, F. Wang and A. Hu, Chin. J. Catal., 2008, 29, 957.

12 H. Wu, F. Zhang, Y. Wan and L. Ye, Lett. Org. Chem., 2008, 5, 209.

13 Z. Qiu and J. Texter, Curr. Opin. Colloid Interface Sci., 2008, 13, 252.

14 G. Singh, G. Singh and T. S. Kang, J. Phys. Chem. B, 2016, 120, 1092-1105.

15 Z. Miskolczy, K. Sebok-Nagy, L. Biczok and S. Gokturk, Chem. Phys. Lett., 2004, 400, 296.

16 A. Modaressi, H. Sifaoui, M. Mielcarz, U. Domanska and M. Rogalski, Colloids Surf., A, 2007, 302, 181.

17 B. L. Bhargava and M. L. Klein, Mol. Phys., 2009, 107, 393.

18 K. S. Rao, P. S. Gehlot, T. J. Trivedi and A. Kumar, J. Colloid Interface Sci., 2014, 428(15), 267-275.

19 M. D. F. Pereira, R. Chevrot, E. Rosenfeld, V. Thiery and T. Besson, J. Enzyme Inhib. Med. Chem., 2007, 22, 577.

20 B. Halova-Lajoie, W. Brumas, M. M. L. Fiallo and G. Berthon, J. Inorg. Biochem., 2006, 100, 362.

21 D. N. Garad and S. B. Mhaske, J. Org. Chem., 2017, 82, 10470.

22 M. Dabiri, L. N. Farajinia, M. S. Kazemi and H. R. Khavasi, Org. Biomol. Chem., 2017, 15, 6264.

23 M. M. Wang, G. L. Dou and D. Q. Shi, J. Comb. Chem., 2010, 12, 582 .

24 X. S. Wang, K. Yang, J. Zhou and S. J. Tu, J. Comb. Chem., 2010, 12, 417.

25 X. Liu, H. Fu, Y. Jiang and Y. Zhao, Angew. Chem., Int. Ed., 2009, 48, 348.

26 S. M. Roopan, T. Maiyalagan and F. N. Khan, Can. J. Chem., 2008, 86, 1019.

27 J. Li, X. Chen, D. Shi, S. Ma, Q. Li, Q. Zhang and J. Tang, Org. Lett., 2009, 11, 1193.

28 M. Wang, T. Zhang, Y. Liang and J. Gao, Monatsh. Chem., 2012, 143, 835.

29 J. Tang, D. Shi, L. Zhang, Q. Zhang and J. Li, Synth. Commun., 2010, 40, 632.

30 H. B. Klevens, J. Phys. Colloid Chem., 1948, 52, 130.

$31 \mathrm{~T}$. Inoue, H. Ebina, B. Dong and L. Zheng, J. Colloid Interface Sci., 2007, 314, 236.

32 N. B. Vargaftik, B. N. Volkov and L. D. Voljak, J. Phys. Chem. Ref. Data, 1983, 12(3), 817-820. 